\title{
Selective impairment of emotion recognition through music in Parkinson's disease: does it suggest the existence of different networks for music and speech prosody processing?
}

\author{
Tobias A. Mattei ${ }^{1 *}$, Abraham H. Rodriguez ${ }^{2}$ and Juri Bassuner ${ }^{3}$ \\ ${ }^{1}$ Neurosurgery Department, Ohio State University, Columbus, OH, USA \\ ${ }^{2}$ Department of Surgery/Division of Neurosurgery, University of Missouri, Columbia, MO, USA \\ ${ }^{3}$ Department of General Medicine, School of Medicine, University of Missouri, Columbia, MO, USA \\ ${ }^{*}$ Correspondence: tobiasmattei@yahoo.com
}

Edited by:

Teppo Särkämö, University of Helsinki, Finland

Keywords: music therapy, emotion recognition, Parkinson disease, speech prosody, non-motor symptoms, rehabilitation

\section{A commentary on}

Not all sounds sound the same: Parkinson's disease affects differently emotion processing in music and in speech prosody

by Lima, C. F., Garrett, C., and Castro, S. L. (2013). J. Clin. Exp. Neuropsychol. 35, 373-392. doi: 10.1080/13803395.2013. 776518

Although the main hallmark of Parkinson's disease (PD) are its motor symptoms, such as bradykinesia, resting tremor, postural instability, and rigidity, it has been increasingly recognized that such disease constitutes, in fact, a complex degenerative process which presents a variety of different clinical manifestations. Actually, in the last years, significant attention has been devoted to the so-called non-motor symptoms of PD, especially psychiatric ones, such as anxiety, depression, apathy, dysphoria and irritability, which have been shown to be present even in the early stages of disease (Leroi et al., 2012) and which show increasing intensity with its progression. Similarly, sleep disorders (such as difficulty for initiating sleep, frequent night awakenings, nocturia, restless legs syndrome, apnea, parasomnias and increased daytime sleepiness) have already been shown to occur with increased prevalence in patients with $\mathrm{PD}$ in comparison to the healthy population (Raggi et al., 2013). Nevertheless, the literature on the effects of PD upon other higher cognitive features, such as musical processing, is still scarce.
Motivated by such increased attention to non-motor symptoms $\mathrm{PD}$, and with basis on previous physiological studies which have demonstrated that the perception of music rhythm and beat is mediated mainly by the basal ganglia (the major anatomical complex involved in the etiology of PD) (Grahn, 2009), several recent studies have investigated the effects of PD over music perception. For example, it has already been shown that patients with PD have reduced capacity of synchronizing movements to a beat and, in turn, discriminating changes in tempo ("faster" vs. "slower") (Grahn and Brett, 2009). Interestingly, such difficulty has been shown to be more marked when the beat is introduced at a slower tempo and progressively speeded up (McAuley et al., 2012). In such study, however, the capacity of patients with PD of discriminating changes in non "beat-based" rhythms did not significantly differ from that of a healthy control group. Ultimately, by correlating the impairment in beat perception with a decline in the coordinated functional activity between the basal ganglia, thalamus, premotor and supplementary motor regions, such studies have underscored the importance of the motor circuitry in beat perception.

Nevertheless, in a recently published study, Lima et al. (2013) demonstrated that the effects of PD upon music perception may not be limited to rhythm detection, but may also involve the recognition of emotions as expressed through music. The authors of such investigation have demonstrated that patients with PD had increased difficulty to recognize happiness and peacefulness, when combinedly expressed through music lyrics and rhythm, while presenting intact perception of sadness and fear. Comparatively, in relation to non-musical speech, such patients presented only a mild global impairment of emotion recognition which seemed to be more a result of an executive dysfunction that a direct effect of the disease over the limbic system. Similarly, other previous investigations had already demonstrated that a dysfunction in the dopaminergic system may only partially explain the observed impairment in emotion recognition through facial expressions in patients with PD (Bediou et al., 2012), as the treatment with levodopa did not significantly modify such deficit. This finding was further confirmed by a recent meta-analysis on the issue (Gray and Tickle-Degnen, 2010). Conversely, in studies evaluating the effects of surgical treatment for Parkinson disease with deep brain stimulation (DBS), a therapeutic strategy which has been suggested to have broader effects in terms of modulating several interconnected circuits and not only the deep basal ganglia, it was possible to observe a synergistic improvement in the ability of recognizing the emotional content of facial expressions after combined treatment with both DBS and levodopa (Mondillon et al., 2012).

In another study which also demonstrated impaired recognition of emotions as expressed through music in patients with $\mathrm{PD}$, it was possible to observe that, although the observed deficit in fear 
recognition was at least partially associated with some degree of executive dysfunction, the deficit in emotion recognition in patients with PD persisted even after adjusting for executive functioning levels (van Tricht et al., 2010). Additionally, despite the fact that a previous study has suggested that depressed patients may have a negative emotional bias when evaluating musical stimuli (Punkanen et al., 2011), in this study the observed deficit in emotion recognition through music was not related to depressive symptoms, disease duration or severity of motor symptoms. All these results suggest that, although the observed impairment of emotion recognition through music in patients with PD may be partially related to a certain degree of executive dysfunction, it most likely reflect a separate primary cognitive deficit in emotional processing.

The suggestion that it may be possible to localize specific cognitive features of emotional and music processing to single anatomical areas of the brain has been investigated by some recent studies which evaluated the neuroanatomical regions activated by facial and music recognition in patients with semantic dementia and Alzheimer's disease (Omar et al., 2011). The results of such investigations demonstrated that the neurocognitive process involved in emotion recognition through facial expressions and music seems to be mediated by different but interconnected networks, with specific patterns of activation depending on the familiarity of the presented sensorial stimuli. In practical terms, the anterior tip of the right anterior temporal lobe seems to directly correlated with the ability to recognize famous tunes and famous faces, while the ability of identifying everyday tunes seems to activate the right mesial temporal structures, especially the amygdala (Hsieh et al., 2011).

In opposition to the presented evidence which support the thesis that music and speech prosody may be mediated by different neurophysiological networks, several previous investigations have demonstrated a very close relationship between music perception and prosody (Thompson et al., 2004; Hutchins et al., 2010; Escoffier et al., 2013). Therefore, the verification of isolated effects of PD upon emotional processing through music perception (but not prosody) may consist in either a bias of the employed methodological approach, or an isolated effect related to motorrelated rhythm dysfunction in patients with PD, rather than a true evidence of the existence of distinct networks for processing emotions (one musical and one non-musical) in normal physiological situations. Ultimately, further clinical and imaging studies are still required in order to confirm the validity of generalizing such findings in patients with PD to the healthy population.

Finally, from a methodological standpoint, the findings of Lima et al. study suggest that future attempts of investigating the effects of degenerative diseases (as well as their treatment) upon the cognitive psychology of emotion processing should focus on specific neuropsychological domains (Hsieh et al., 2012), as there might be significant differences among the effects of such diseases upon recognition of emotional content from specific sensory inputs (for example, through visual or auditory modalities), and even among specific variations within one specific sensory modality (such as the difference between emotional recognition through musical and non-musical speech).

\section{REFERENCES}

Bediou, B., Brunelin, J., d'Amato, T., Fecteau, S., Saoud, M., Hénaff, M. A., et al. (2012). A comparison of facial emotion processing in neurological and psychiatric conditions. Front. Psychol. 3:98. doi: 10.3389/fpsyg.2012.00098

Escoffier, N., Zhong, J., Schirmer, A., and Qiu, A. (2013). Emotional expressions in voice and music: same code, same effect. Hum. Brain Mapp. 34, 1796-1810. doi: 10.1002/hbm.22029

Grahn, J. A. (2009). The role of the basal ganglia in beat perception: neuroimaging and neuropsychological investigations. Ann. N.Y. Acad. Sci. 1169, 35-45. doi: 10.1111/j.1749-6632.2009.04553.x

Grahn, J. A., and Brett, M. (2009). Impairment of beat-based rhythm discrimination in Parkinson's disease. Cortex 45, 54-61. doi: 10.1016/j.cortex.2008.01.005

Gray, H. M., and Tickle-Degnen, L. (2010). A metaanalysis of performance on emotion recognition tasks in Parkinson's disease. Neuropsychology 24, 176-191. doi: 10.1037/a0018104

Hsieh, S., Hornberger, M., Piguet, O., and Hodges, J. R. (2011). Neural basis of music knowledge: evidence from the dementias. Brain J. Neurol. 134, 2523-2534. doi: 10.1093/brain/awr190

Hsieh, S., Hornberger, M., Piguet, O., and Hodges, J. R. (2012). Brain correlates of musical and facial emotion recognition: evidence from the dementias. Neuropsychologia 50, 1814-1822. doi: 10.1016/j.neuropsychologia.2012.04.006

Hutchins, S., Gosselin, N., and Peretz, I. (2010). Identification of changes along a continuum of speech intonation is impaired in congenital amusia. Front. Psychol. 1:236. doi: 10.3389/fpsyg.2010.00236

Leroi, I., Pantula, H., McDonald, K., and Harbishettar, V. (2012). Neuropsychiatric symptoms in Parkinson's disease with mild cognitive impairment and dementia. Parkinsons Dis. 2012, 308097. doi: 10.1155/2012/308097

Lima, C. F., Garrett, C., and Castro, S. L. (2013). Not all sounds sound the same: parkinson's disease affects differently emotion processing in music and in speech prosody. J. Clin. Exp. Neuropsychol. 35 , 373-392. doi: 10.1080/13803395.2013. 776518

McAuley, J. D., Henry, M. J., and Tkach, J. (2012). Tempo mediates the involvement of motor areas in beat perception. Ann. N.Y. Acad. Sci. 1252, 77-84. doi: 10.1111/j.1749-6632.2011.06433.x

Mondillon, L., Mermillod, M., Musca, S. C., Rieu, I., Vidal, T., Chambres, P., et al. (2012). The combined effect of subthalamic nuclei deep brain stimulation and L-dopa increases emotion recognition in Parkinson's disease. Neuropsychologia 50, 2869-2879. doi: 10.1016/j.neuropsychologia.2012. 08.016

Omar, R., Henley, S. M., Bartlett, J. W., Hailstone, J. C., Gordon, E., Sauter, D. A., et al. (2011). The structural neuroanatomy of music emotion recognition: evidence from frontotemporal lobar degeneration. Neuroimage 56, 1814-1821. doi: 10.1016/ j.neuroimage.2011.03.002

Punkanen, M., Eerola, T., and Erkkilä, J. (2011). Biased emotional recognition in depression: perception of emotions in music by depressed patients. J. Affect. Disord. 130, 118-126. doi: 10.1016/j.jad.2010.10.034

Raggi, A., Bella, R., Pennisi, G., Neri, W., and Ferri, R. (2013). Sleep disorders in Parkinson's disease: a narrative review of the literature. Rev. Neurosci. 24, 279-291. doi: 10.1515/revneuro-2013-0002

Thompson, W. F., Schellenberg, E. G., and Husain, G. (2004). Decoding speech prosody: do music lessons help. Emotion 4, 46-64. doi: 10.1037/15283542.4.1.46

van Tricht, M. J., Smeding, H. M., Speelman, J. D., and Schmand, B. A. (2010). Impaired emotion recognition in music in Parkinson's disease. Brain Cogn. 74, 58-65. doi: 10.1016/j.bandc.2010.06.005

Received: 16 July 2013; accepted: 20 August 2013; published online: 12 September 2013.

Citation: Mattei TA, Rodriguez AH and Bassuner J (2013) Selective impairment of emotion recognition through music in Parkinson's disease: does it suggest the existence of different networks for music and speech prosody processing? Front. Neurosci. 7:161. doi: 10.3389/fnins.2013.00161

This article was submitted to Auditory Cognitive Neuroscience, a section of the journal Frontiers in Neuroscience.

Copyright (c) 2013 Mattei, Rodriguez and Bassuner. This is an open-access article distributed under the terms of the Creative Commons Attribution License (CC BY). The use, distribution or reproduction in other forums is permitted, provided the original author(s) or licensor are credited and that the original publication in this journal is cited, in accordance with accepted academic practice. No use, distribution or reproduction is permitted which does not comply with these terms. 